\title{
CLASSIFICATION ERRORS IN LOW-DISPERSION SPECTROSCOPY
}

A. Bruch, M. Buescher, W. Samson and W. C. Seitter

Astronomisches Institut der Universität Milnster

With the two parts of the Bonn Spectral Atlas it was hoped to provide a tool for the improvement of accuracy in low-dispersion spectral classification. Earlier estimates from our spectra by different observers with classification experience indicated a rather small increase in classification errors with decreasing resolution. They amount to 0.17 spectral types per $1000 \AA \mathrm{mm}^{-1}$ increase (larger numbers) in dispersion in the range $200-1300 \AA \mathrm{mm}^{-1}$ dispersion added to the 0.1 spectral types which are typical errors at MK classification dispersion near $100 \AA \mathrm{mm}^{-1}$ and 0.56 luminosity classes per $1000 \AA \mathrm{mm}^{-1}$ increase (1arger numbers) in dispersion in the range $200-1300 \AA \mathrm{mm}^{-1}$ dispersion added to the 0.6 luminosity classes which are typical errors at MK classifications near $100 \AA \mathrm{mm}^{-1}$. In order to improve on the error determinations the three first-named authors, all graduate students with no previous experience in spectral classification, classified independently between 800 and 1300 spectra with each of the three Bonn Atlas dispersions of 240,645 and 1280 $\AA \mathrm{mm}^{-1}$. The classification errors of the three students differ by very small amounts and thus are averaged in the following discussion.

of the various error determinations we present the following:

1. Average values of absolute differences between student classifications and MK standards. These values may be influenced by an unknown recogniton factor because the plates used for classification were largely the same as those obtained for the atlas plates. Furthermore the number of known MK standards on the Schmidt-telescope plates was not much larger than the number of standards used in the atlas.

2. Average values of internal deviations from the mean between any two student classifications. This group contains between 15 and 
37\% MK standards for which the reservations concerning the recognition factor also apply. The errors are thus still somewhat underestimated.

3. Average values of internal deviations from the mean between any two student classifications corrected for the recognition of standard stars. In this group all standard stars are considered to be reclassified with zero error. The errors of the samples are then increased by the factor (total number of stars/ number of stars with classification errors). The errors thus determined do not contain systematic deviations from the standard stars. Graphic presentations, however, show that these must be small.

4. The errors given are determined in the following way: the mean absolute difference Cannon-standards was determined and then used as a systematic correction factor applied to the mean absolute difference Cannon-students. The results are considered upper limits to the differences students-standards.

The same procedures (except, of course, 4) were applied to obtain the various errors in luminosity class. Instead of 4 a conversion factor obtained by comparing the entries of 1 and 4 in Table I were applied to the values of 1 in Table II in order to obtain 4.

Within the observed uncertainties the classification errors of the three different dispersions are sufficiently similar to permit the following general statements. At dispersions $200-1300 \mathrm{Amn}-1$ MK classification is possible with errors which are at best of the same order as those found at MK. dispersions and at worst larger by factors 2 - 3 . These accuracies can be obtained by inexperienced classifiers with no other tool than the Bonn Spectral Atlas. It is suggested that MK classification should be attempted at all dispersions in place of the so-called natural groups which do not fully employ the information content of low-dispersion spectra.

TABLE I

ERRORS IN SPECTRAL CLASS

\begin{tabular}{|cccc|}
\hline pispersion, A m -1 & 240 & 645 & 1280 \\
\hline No & & & \\
1 & 0.15 & 0.19 & 0.19 \\
2 & 0.08 & 0.10 & 0.09 \\
3 & 0.10 & 0.13 & 0.12 \\
4 & 0.21 & 0.22 & 0.25 \\
\hline
\end{tabular}


TABLE II

ERRORS IN LUMINOSITY CLASS

\begin{tabular}{|cccc|}
\hline \hline Di.spersion, A m-1 & 240 & 645 & 1280 \\
\hline No & & & \\
1 & 1.2 & 1.5 & 1.4 \\
2 & 0.6 & 0.6 & 0.7 \\
3 & 0.7 & 0.7 & 1.0 \\
4 & 1.7 & 1.7 & 1.8 \\
\hline
\end{tabular}

